disposal. Sustainability and monetary incentives were the main reported driving factors for recycling engagement, and all pharmacies would consider subscribing to a recycling scheme if available.

Conclusions Despite interest from local pharmacies, there are no available inhaler recycling services in the area we examined, and safe disposal uptake is very low. Promotion, patient education and investment are required for the NHS to meet its sustainability targets.

\section{P157 EFFECTIVENESS OF A MULTI-DISCIPLINARY COMMUNITY RESPIRATORY TEAM DURING THE COVID-19 PANDEMIC}

${ }^{1} \mathrm{E}$ Johnson, ${ }^{1} \mathrm{E}$ Turner, ${ }^{1} \mathrm{~S}$ Gingles, ${ }^{2} \mathrm{~K}$ Levin, ${ }^{3} \mathrm{E}$ Mackay, ${ }^{3} \mathrm{C}$ Roux, ${ }^{3} \mathrm{M}$ Milligan, ${ }^{3} \mathrm{M}$ Mackie, ${ }^{3} \mathrm{~K}$ Farrell, ${ }^{3} \mathrm{~K}$ Murray, ${ }^{3} \mathrm{~S}$ Adams, ${ }^{3} \mathrm{~J}$ Brand, ${ }^{1} \mathrm{D}$ Anderson, ${ }^{4} \mathrm{H}$ Bayes. ${ }^{1}$ Department of Respiratory Medicine, Queen Elizabeth University Hospital, NHS Greater Glasgow \& Clyde, Glasgow, UK; ${ }^{2}$ Health Services, NHS Greater Glasgow \& Clyde, Glasgow, UK; ${ }^{3}$ NHS Greater Glasgow and Clyde Community Respiratory Response Team, Glasgow, UK; ${ }^{4}$ Department of Respiratory Medicine, Glasgow Royal Infirmary, NHS Greater Glasgow \& Clyde, Glasgow, UK

\subsection{6/thorax-2021-BTSabstracts.266}

Introduction The Community Respiratory Response Team (CRRT) was established to manage patients within Greater Glasgow \& Clyde NHS Trust (NHS GGC) with chronic lung disease at home during the COVID-19 pandemic. We analysed the effectiveness of a triage pathway for appropriately targeting care, and overall effectiveness of the service in reducing the outcomes of Emergency department (ED) attendance, hospital admission and death.

Methods Electronic health records of patients referred in May 2020 were retrospectively reviewed. The relationship between CRRT triage pathway and emergency department (ED) attendance, hospital admission and death within 28 days of referral was assessed, with respect to primary respiratory condition.

Results Mean patient age was 69 years (median 71; IQR 6279). 66\% of patients were female. Figure 1 shows CRRT patient triage and outcomes. Excepting the blue 'end of life care' triage pathway, higher triage category was associated with higher rates of $\mathrm{ED}$ attendance, hospital admission and death. The only death in the green triage group was due to a non-respiratory cause. Patients triaged red or amber were more likely to receive more than one consultation. In particular, patients with COPD in red and amber triage groups were more likely to have multiple CRRT consultations or a home visit.

$87 \%$ of consultations were conducted remotely; mean 4.4 consultations/patient; $35 \%$ received a home visit. No nosocomial COVID-19 infections occurred. 52\% of deaths occurred in patients with COPD or asthma/COPD overlap. Increasing number of consultations was associated with reduced mortality but not reduced ED attendance or hospital admissions. However, for patients diagnosed with COPD and triaged as highest risk, having over 3 consultations was associated with lower ED attendance $(16 \%$ vs $30 \%)$ and admission rates $(18 \%$ vs 26\%). Hospital admissions and inpatient deaths for COPD patients in the 2nd quarter of 2020 were $47 \%$ and $65 \%$ of previous years, respectively.

Conclusions The NHS GGC CRRT was able to safely and appropriately risk stratify patients and complement tertiary care by providing support at home with potential impact on reducing hospital admissions and deaths. Wider implementation of multidisciplinary community respiratory care could benefit patients and the healthcare service.

\section{P158 THORACIC ULTRASOUND ON THE RESPIRATORY POST- TAKE WARD ROUND: ASSESSING THE IMPACT ON CLINICAL DECISION-MAKING AND THE PATIENT JOURNEY}

${ }^{1} \mathrm{M}$ Steward, ${ }^{2} \mathrm{HG}$ Bakere, ${ }^{1} \mathrm{TG}$ Burden. ${ }^{1}$ Royal Devon and Exeter NHS Foundation Trust, Exeter, UK; ${ }^{2}$ Royal Cornwall Hospitals NHS Trust, Truro, UK

\subsection{6/thorax-2021-BTSabstracts.267}

Introduction Thoracic ultrasound (TUS) has become indispensable when assessing the acutely unwell respiratory patient. We examined the impact of TUS on clinical decision-making and patient management, inviting discussion regarding the routine use of TUS on the respiratory post-take ward round (PTWR). Methods Data was collected prospectively from fifty consecutive patients allocated to the acute respiratory PTWR. TUS

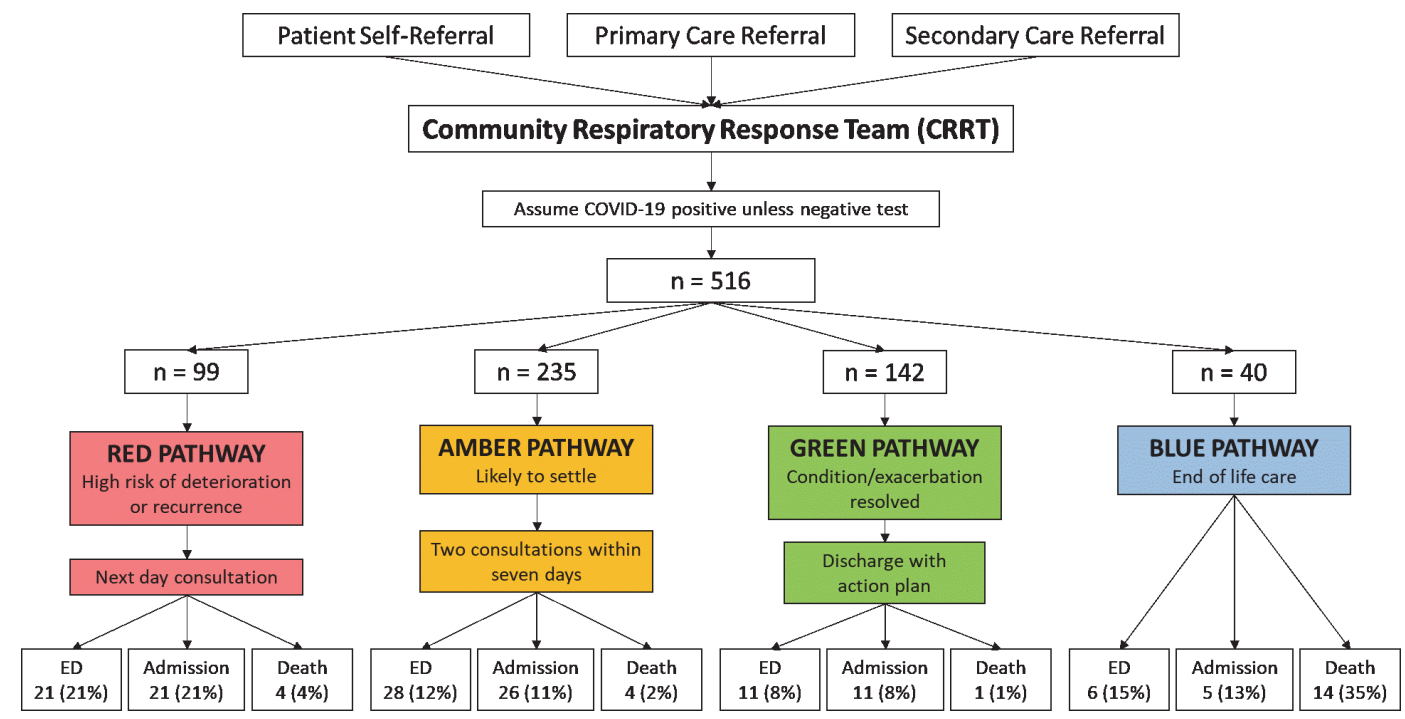

Abstract P157 Figure 1 
was performed and standardised utilising the validated 'BLUE protocol', performed by the same operator. Domains included demographics, respiratory comorbidities, diagnoses, and management plans prior to, and following TUS.

Results Of fifty patients scanned, TUS altered overall management in 22 (44\%). Primary diagnosis was changed in $26 \%$, treatments in $34 \%$, investigations in $28 \%$, and all three aspects in $18 \%$. TUS performed well in fluid balance decisions and identifying lung consolidation.

Patient groups where TUS would not alter management were identified, with reduced odds seen with pre-existing airways disease (odds ratio (OR) $0.37,95 \%$ confidence interval (CI) 0.12-1.17), and in patients with airways disease and wheeze on auscultation (OR 0.08, 95\%, CI 0.01-0.77).

Chest $x$-ray (CXR) reports differed from TUS findings in 12 (24\%). 21 (42\%) patients later underwent computed tomography (CT) examination with CT reports corresponding with positive TUS findings in $100 \%$, with no further emendation of diagnoses (excluding incidental findings). Data was not collected to assess the time implications of performing ultrasound on the consultation, and we acknowledge that not all Respiratory physicians are ultrasound trained thus limiting the provision of thoracic ultrasound.

Discussion The use of TUS impacted significantly on decisionmaking on the Respiratory PTWR. Unnecessary radiology requests, ionising radiation, and cost were avoided. Within the constraints of the study group, TUS seems less useful when assessing patients with pre-existing airways disease. TUS has excellent correlation with CT findings, outperforms CXR, appearing to offer a comprehensive, streamlined respiratory assessment at the 'front-door'.

With ultrasound becoming more accessible to clinicians, and with increasing demands on CT departments, we welcome discussion regarding regular use of TUS on the PTWR. Further data would be desirable to assess whether its use early in admission is correlated with a reduced length of stay and improved patient outcomes.

\section{P159 OUTCOME FROM INVASIVE VENTILATION FOR PATIENTS WITH LEARNING DISABILITY}

D McCourt, HM Tedd, TB Fretwell, T Doris, PB Messer. Newcastle Hospitals, Newcastle Upon Tyne, UK

\subsection{6/thorax-2021-BTSabstracts.268}

The annual learning disability mortality review (LeDeR) ${ }^{1}$ highlights that patients with learning disability (LD) are twice as likely to die of avoidable causes, with inappropriate ceilings of treatment being instigated in patients with LD: 23\% of 'Do Not Attempt Cardiopulmonary Resuscitation' forms documented inappropriate medical conditions such as a learning disability as the sole reason to not attempt resuscitation $^{1}$. The COVID19 pandemic has further highlighted this issue, with people with LD being at 6 times the risk of death from COVID19 than those without LD; people with LD aged 18-34 were 30 times more likely to die of COVID 19. ${ }^{2}$ However, there is little data out there to help support decision making around invasive ventilation in people with LD.

We aimed to explore the outcomes of patients with LD admitted to a single critical care unit for invasive ventilation
Methods We reviewed the notes of patients with learning disability requiring intubation and ventilation over a 5-year period (2016-2020). Data was retrospectively collected on survival, rates of tracheostomy insertion and requirement for long term ventilation (LTV) or cough augmentation.

Results 15 patients were identified with LD who required invasive ventilation. 93\% survived critical care admission. 7\% required tracheostomy with subsequent decannulation, whilst a further 7\% required long term tracheostomy. 40\% were commenced on LTV. 46\% required long term cough augmentation via cough assist device. 46\% have subsequently died, with a mean survival following critical care admission of 2.5 years. Mean duration from invasive ventilation in the survivor group is 5.5 years.

Conclusion We have demonstrated good outcomes in patients with LD admitted to critical care for invasive ventilation. Although there was a high requirement for LTV and cough augmentation following admission to critical care for invasive ventilation, we have demonstrated both good survival to discharge from critical care and good long term survival in this group. This small single centre study highlights the need for further research to aid decision making around escalation decisions in patients with LD.

\section{REFERENCES}

1. The learning disability mortality review (LeDeR): Annual report. 2020.

2. COVID19 deaths of people identified as having learning disabilities: summary. Public Health England. 2020.

\section{P160 COMMUNITY RESPIRATORY STAFF IN-REACH INTO CARE HOMES FINDS UNMET NEED AND ALLOWS OPTIMIZATION OF PATIENT CARE PLUS CARE HOME STAFF EDUCATION}

HS Hill, K Keeling, I Young, J Chandler, MCP Apps. North East London Foundation NHS Trust, Billericay, Essex, UK

\subsection{6/thorax-2021-BTSabstracts.269}

Introduction During the Covid epidemic the care of patients in care homes to optimize their therapy and prevent admission has become a national Priority. In the Basildon and Brentwood CCG area staff normally working in the community respiratory service were redeployed to visit all the care homes in the area and review all patients with respiratory disease to optimize therapy, advise flu immunisation and support their care.

Methods All care homes in the area of Basildon, Brentwood, Billericay and Wickford were visited and a review carried out on all patients resident there with respiratory disease. This included reviewing both notes and patients to find patients with respiratory disease. Therapy was reviewed and optimised, including obtaining new inhalers, spacer devices and rescue packs.

Results 163 patients were reviewed, of whom 116 had evidence of respiratory disease. 31 of these were already known to the community respiratory services and 85 were not known. $75 \%$ of patients needed a new salbutamol inhaler or aero-chamber spacer device. 58\% of patients did not have a rescue pack of antibiotics and steroids and this was provided. $85 \%$ of those not already known to the service required salbutamol,. A spacer and a rescue pack.

These were all provided. 